\title{
Take a breath: Breathing Biofeedback Relaxation Intervention for Wheelchair Users in City Navigation
}

\author{
Roxana Ramirez Herrera, Catherine Holloway, Dafne Zuleima Morgado Ramirez, Bingqing Zhang, \\ Youngjun Cho
}

\begin{abstract}
Manual wheelchair users experience numerous invisible barriers while navigating cities, often reporting how stressful journeys are. This stress affects a wheelchair user's quality of life. To alleviate such psychological burden, we propose a novel intervention strategy with a respiratory biofeedback interface which is designed to help users feel relaxed in urban navigation. We conduct a study in a real-world setting to explore its potential to provide real-time psychological support. From qualitative and quantitative analysis, we report on strengths and weaknesses of the approach.
\end{abstract}

\section{INTRODUCTION}

Propelling a wheelchair in a city is hard work. Over $50 \%$ of wheelchair users will develop upper limb injury and associated pain due in part to the high and repetitive push forces needed to propel [1][2]. The difficulty of navigating in a city is not only a physical experience, but also cognitive and emotional [3]. For example, wheelchair users using public transport have reported social barriers which lead directly to anxiety and frustration and later to fear of travelling[4].

Improving accessibility of the built environment and public transport could be the most obvious answers to decrease the physical and mental stressors of wheelchair users. However, achieving this requires a long-term effort that involves a wide variety of stakeholders - spanning urban planners to government; transport providers to building operators leaving wheelchair users with a lack of support at this very moment.

Biofeedback techniques - which measure an individual's physiological data to provide feedback - have been used as efficacious psychological tools to assist relaxation and stress management [5]. For example, [6] has been designed to help city commuters relax in the car with guided slow breathing.

There is a small body of literature on technological interventions specifically targeted for psychological support for wheelchair users. One study tested a mindfulness mobile app and evaluated the effects of a long-term mindfulness training programme with wheelchair basketball players to understand the impact of this practice on athletic performance during competition [7]. The study did not investigate the effect on activities of daily living. Moreover, the psychological burdens of wheelchair users while performing daily stressful activities such as urban navigation has been unexplored. To address this, we focus on devising a technological relaxation intervention that can enable wheelchair users to cope better with their daily stressful

All authors are with the Computer Science Department, University College London, Gower St, London,WC1E6BT. (e-mail: ucesrr0@ucl.ac.uk). journeys. In this paper, we report on a proof-of-concept study. Our objectives are: 1) to build and evaluate a relaxation intervention which harnesses breathing biofeedback for inexperienced wheelchair users during a representative journey in a city; 2) To develop an understanding of the strengths and weaknesses of our proposed design; 3) to provide design implications for future development.

\section{THE BREATHING AUDIO FEEDBACK INTERFACE}

\section{A. Background}

Yu et al [5] identified three key components of a biofeedback system: (1) the type of intervention (technique used to selfregulate), (2) the biofeedback display (presentation methods) and (3) the biofeedback modality (physiological signals). These should be carefully chosen, taking into consideration the nature of the task and the context in which the system will be used. In this paper, we consider mobility through manual wheelchair propulsion as the main task and urban navigation as the context.

1) Type of intervention. Self-regulation when using biofeedback is accomplished by implementation of relaxation techniques such as controlled breathing, muscle relaxation, positive imagery or meditation [8]. The biofeedback tools can be used to train self-regulation skills or improve resilience to stressful situations or tasks whilst they are taking place (i.e. daily hassles) [5].

Breathing training consists of controlling respiratory and/or tidal volume rate, with the aim to keep a breathing rate of between $4-10$ breaths per minute (bpm) [9]. Breathing patterns in this range are referred to as slow breathing. Positive imagery can also be used and consists of visualization of images that elicit relaxation, while muscle relaxation involves alternating between tensing and relaxing muscles to relief anxiety [8]. These last two however, would not be suitable for our context. Visual attention needs to be on the changing urban environment, while muscle relaxation is not possible when upper body muscles are engaged on wheelchair propulsion.

We have chosen regulation of breathing, as it is one of the most used methods to control biofeedback signals. Breathing control has the additional benefit of being intuitive and easy to learn [8]. Furthermore, evidence of its effectiveness in mental well-being has been extensively explored in the literature [9].

R. Ramirez Herrera is partly supported by a scholarship from the National Council of Science and Technology in Mexico (CONACYT). 


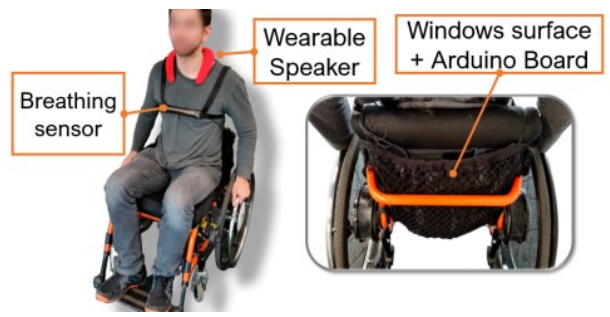

Figure 1. Breathing biofeedback interface for wheelchair users

2) Biofeedback display. Amongst the feedback modalities visual, haptic and auditory - the most common is visual feedback, typically presented on a PC or mobile screen [10].Visual feedback however is not suitable in our context, as staring at screens is a distraction that can become a safety hazard when navigating the city [11]. Likewise, haptic feedback has drawbacks. In the case of wheelchair propulsion, wheels in contact with the pavement can interfere with haptic perception, as described by Eriksson et al [12].

Another downside of haptic feedback comes from users' preferences. For instance, in [13], respiration haptic feedback was rated significantly lower in enjoyability and usefulness when compared to audio and visual feedback, with $75 \%$ participants expressing open negative reviews of the first.

Given this, we chose auditory feedback for our intervention. Its use in breathing regulation has been widely accepted and implemented, with Yokoyama et al identifying its convenience if wanting to combine it with work or other activity [14]. Sound is versatile and can be presented in a variety of forms, from simple cues and warnings, to musical interventions [15], or naturalistic sounds [16].

3) Biofeedback modality. This refers to the physiological variable being measured. Heart rate variability (HRV) biofeedback has been the most commonly used, and is normally measured through electrocardiogram or blood volume pulse [5][17]. However, most of its sensor forms might interfere with wheelchair propulsion. For instance, PPG/BVP sensors on a finger or wrist form factor could interfere with adequate wheelchair hand rim grip, and users have reported discomfort during propulsion [18]. In addition, representation of HRV is typically in visual form [5], which is unacceptable for our context. Given this, we use a chest belt-based breathing sensor that does not interfere with the task whilst providing acceptable signal mapping to the output.

\section{B. Prototype implementation}

Figure 1 shows our proposed interface. This provides sound feedback to the users based on their breathing rate, whilst still allowing wheelchair users to self-propel naturally in urban settings. This interface has been inspired by the portable breathing sound interface in [15][19]. Although [15][19] use 2 belts, one on the chest and one on the abdomen, we chose to use just the one on the chest to decrease obtrusiveness. Given that the thoracic muscles are involved during wheelchair propulsion, the simple adjustable belt would tend to slip down. Therefore, we added two adjustable overshoulder suspenders to keep the belt in place.

The chest belt consists of an adjustable band with the middle front part made of a conductive material that stretches and changes resistance in proportion to the expansion and contraction of the chest, thus working as a respiratory sensor. The voltage signal is read by a microcontroller (Arduino MKR1000) which communicates via serial port to a Windows Surface. Both microcontroller and Windows Surface are safely enclosed in a thin and lightweight bag attached to the back of the chair. The Windows Surface executes a Python script that 'sonifies' the user's breathing with white noise. For our interface, we map the pitch of the white noise to the respiration amplitude as measured by the respiration sensor by using (1):

$$
\text { Pitch coefficient }=1-0.95 \frac{\left(\mathrm{x}_{\text {last }}-\mathrm{x}_{\min }\right)}{\left(\mathrm{x}_{\max }-\mathrm{x}_{\min }\right)}
$$

Where $\mathrm{x}$ is a vector which size corresponds to the buffering window, $\mathrm{x}_{\text {last }}$ to the last of the samples, and $\mathrm{x}_{\min }$ and $\mathrm{x}_{\max }$ to the local minima and local maxima. Pitch will then increase with inspiration until saturation and will decrease with exhalation. In the case of the white noise, increasing the pitch produces a sensation of 'loudness' and decreasing it of 'quietness', with a full breathing cycle being reported to evoke the sound of the ocean waves or rainfall [13]. This effect is a desirable feature in biofeedback, as ecological sounds have been shown to be better accepted than artificial ones [16].

The sampling rate of the breathing signal was determined empirically, by sampling every $5 \mathrm{~Hz}$, from $10 \mathrm{~Hz}$ to $50 \mathrm{~Hz}$. The chosen sampling rate of $20 \mathrm{~Hz}$ was the one which produced a feedback that was perceived as more pleasant and the one in which delay between input and output was less noticeable, as evaluated on a pilot test with one volunteer. The signal was filtered in real-time with a moving average filter over every 20 buffered samples.

Most auditory feedback breathing interventions deliver the sound through headphones or by using the speakers of a mobile phone [5]. In an outdoor context however, sound from the mobile phone would get dissipated, while the use of headphones is discouraged as it can pose a safety risk in environments with moving vehicles [20]. We chose to use a wearable speaker that goes around the neck, which canalizes the sound towards the user's ears at a clear volume yet allows to hear the surroundings. These speakers connect via Bluetooth to the Windows Surface.

It may be the case for users to lose attention on the sound feedback whenever they are engaged on a physical activity. Therefore, in our interface, when the breathing rate is measured to be above $10 \mathrm{bpm}$, which is the upper threshold of slow breathing, a warning triggers saying: 'Please remember to breathe slowly'. The warning is only triggered every minute and only if for the past minute, the user had consistently presented a breathing rate of more than $10 \mathrm{bpm}$. (Code: https://github.com/akaimody123/Breathing_belt).

\section{EXPERIMENTAL PROTOCOL AND PARTICIPANTS}

The study received UCL Ethics Committee Approval: UCLIC/1617/025/Staff Holloway/Ramirez. We recruited 12 non-disabled participants (Labelled P1 - P12), 5 males and 7 females, with mean age $31.20 \pm 9.37$ years. All participants used a standard manual wheelchair Quickie Life R fitted with M24 Alber Twion power assist wheels, which were switched 
on to make sure that participants could complete the whole experimental session. At the beginning of each session, participants were given a 20 to $30 \mathrm{~min}$ introduction on how to use a manual wheelchair, based on instruction suggestions adapted from the Wheelchair Skills Training Manual [21]. The training session took part in an open space and the experiment started once participants felt comfortable using the wheelchair.

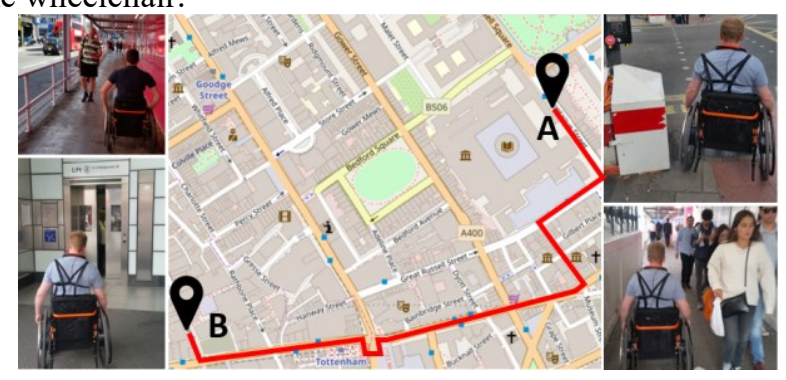

Figure 2: Route followed in Central London

Participants were asked to self-propel the manual wheelchair throughout the streets of central London, following a fixed route from A to B (see Figure 2) of approximately $1200 \mathrm{~m}$ and then to return from $B$ to $A$. The route was chosen as it contained several known barriers (see Table 1). The route took an average of $25 \mathrm{~min}$ to complete. The duration of the route was selected as to be long enough to contain all representative stressors and make participants engage in the task, but not too long to provoke fatigue. The researcher accompanied the participant to give directions and ensure safety, but participants were discouraged from interacting with the researcher.

When travelling in one direction, the participant used the technological intervention, and then on the return journey the participant would wheel without the intervention. The order in which the biofeedback intervention was used was randomized and balanced, i.e. half the participants started by using the feedback and then returned without using it and half started without but used the technological intervention on the return journey. Participants were given a ten-minute rest between journeys.

In preparation for the journey that included biofeedback, participants were also asked to watch a four-minute video on breathing control training, while listening to the prototype's auditory biofeedback. This video was created by the lead researcher and it instructed the participants to synchronize their breathing rate (and in consequence the sounds heard as feedback) with a sinusoidal wave which had a frequency of 6 bpm. After training, participants were reminded to try and keep the breathing rate they had just learned throughout the following journey. The training session was given at a location close to the starting point of the journey, which was considered to be quiet enough to allow for concentration on the video.

The time of the sessions was carefully selected to avoid London's rush hour, which could have increased the risk of accidents, given the crowds and traffic characteristic of peak times in the city. At the end of each session, participants were asked to fill in a questionnaire in which they rated how relaxed they felt after each session (with and without feedback) using the $10 \mathrm{~cm}$ visual analog scale (VAS) from 0 to 10 , with 0 being 'Not at all' and 10 'Very much', which was adapted from the Instant Stress detection questionnaire [22]. Participants were also asked open-ended questions to provide feedback on the technological intervention.

For the quantitative analysis, a Shapiro-Wilks test was used to assess normality of the data. This was followed by a paired t-test analysis to evaluate whether there was a significant difference between the means of the VAS for relaxation in both conditions: with and without the biofeedback system. For the qualitative data, we transcribed the comments of the participants, classified them depending on whether they were considered as qualities of the system or areas of opportunity, and reported them.

TABLE I. BARRIERS ENCOUNTERED THROUGHOUT THE SELECTED ROUTE

\begin{tabular}{|l|l|}
\hline Barriers & \multicolumn{1}{|c|}{ Description and examples } \\
\hline $\begin{array}{l}\text { Sidewalk } \\
\text { obstacles }\end{array}$ & $\begin{array}{l}\text { The smaller streets of the route were chosen to include sidewalk } \\
\text { obstacles. These were found at several points and included } \\
\text { restaurant sign stands, outdoor chairs and tables, and shop } \\
\text { display stands. In addition, participants found unpredictable } \\
\text { obstacles, such as cones or incorrectly parked bicycles. }\end{array}$ \\
\hline $\begin{array}{l}\text { Road } \\
\text { crossings }\end{array}$ & $\begin{array}{l}\text { Participants performed } 6 \text { crossings without traffic lights, and } 7 \\
\text { crossings with traffic lights. }\end{array}$ \\
\hline $\begin{array}{l}\text { Under- } \\
\text { ground } \\
\text { station }\end{array}$ & $\begin{array}{l}\text { Participants were asked to enter Exit 2 of Tottenham Court Road } \\
\text { Station (TCRS), one of the busiest stations in London, by } \\
\text { descending to level -1 on a lift. They were then instructed to } \\
\text { navigate through the station and find the second lift to Exit 1. }\end{array}$ \\
\hline Crowds & $\begin{array}{l}\text { Three main points were included to make sure participants will } \\
\text { encounter crowds: a) the crossing at TCRS b) a section of Oxford } \\
\text { St (busiest shopping street in London) and c) the entrance of the } \\
\text { British Museum (most visited museum in the UK). }\end{array}$ \\
\hline $\begin{array}{l}\text { Uneven } \\
\text { terrains }\end{array}$ & $\begin{array}{l}\text { Examples of these include the cobblestone and similar variations } \\
\text { of pavement found around the British Museum, and the tactile } \\
\text { pavement from ramps at most road crossing points. }\end{array}$ \\
\hline $\begin{array}{l}\text { Construc- } \\
\text { tion sites }\end{array}$ & $\begin{array}{l}\text { Participants were asked to navigate by two construction sites. } \\
\text { One of them required to ascend and descend a metallic and } \\
\text { slightly unstable ramp, the second one involved deviating } \\
\text { participants to propel over the road instead of the sidewalk. }\end{array}$ \\
\hline
\end{tabular}

\section{RESUltS}

From the participants' feedback on the technological intervention, we identified strengths of our system as well as areas of improvement. Most of the positive comments were with respect to the interventions' ability to perform what it was expected to do, with seven participants mentioning how the biofeedback helped them to keep focus on their breathing rate. Two participants were particularly pleased with the idea, with P6 writing: "I like the concept, it reminds me a bit of meditation exercise". P12 found that "the breathing feedback was initially distracting but then calming".

P5 was greatly satisfied with the experience:

It definitely made a difference, I was more conscious of my breathing than I would normally would and it made me slow down, despite doing the breathing on the outward journey, and coming back since, I can still feel very relaxed compared to normal. Thanks for the relaxing experience. By contrast, $\mathrm{P} 9$ and $\mathrm{P} 10$ found the sound brought the opposite result: "to me the sound was a bit too much in addition to navigating streets, etc. (P9)."

Participants identified room for improvement of the prototype: a perceived delay between their breathing and the sound feedback (P2, P4, P7 and P10) and comfortability related to the form factor. $\mathrm{P} 4$ and $\mathrm{P} 11$ described the suspender 
straps as uncomfortable and "kept slipping down", as opposed to P9, who commented on the chest belt being comfortable. As for the quantitative analysis, Figure 3 presents the selfreported relaxation score from the VAS-based questionnaire. Mean score for 'no feedback' condition was $4.60 \pm 2.05$ and mean score for 'feedback' condition was $5.05 \pm 2.22$. The differences between the relaxation scores in both conditions (with and without feedback) were normally distributed, as assessed by the Shapiro-Wilk's test $(\mathrm{p}=0.552)$. Figure 3 shows that although there was no significant difference between conditions $(\mathrm{t}(11)=-1.232, \mathrm{p}=0.244)$, there is a moderate increase in mean value of relaxation score when using the biofeedback system.

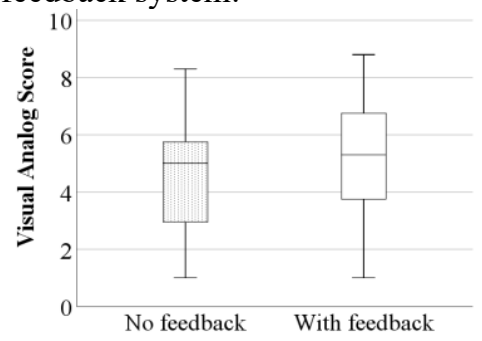

Figure 3: Boxplot of Self-reported relaxation VAS for both conditions

\section{DISCUSSION AND FUTURE WORK}

To the best of our knowledge, this is the first relaxation intervention system for providing real-time physiopsychological support to wheelchair users in urban navigation. It is based on already proven biofeedback techniques and also meets the context specific design criteria and gets further evaluated in situ.

The qualitative responses from participants showed positive feedback on the usability and the positive emotional experience. The lack of statistical significance on the perceived relaxation levels, could be explained by individual differences of the small sample population in self-reporting scores, and the lack of baseline [22]. In addition, even though participants took the same route, and all efforts were made to 'control' for the types of barriers encountered, the urban landscape is constantly changing. Inevitably some of the participants would have been exposed to potentially more stressful situations than others (e.g. closure of one of the lifts from the station). This is part of the complexity of 'in-thewild' studies and it is precisely where this kind of technological interventions need to be evaluated.

A known limitation of our work is that the prototype was evaluated with non-disabled participants. This choice was made based on a risk assessment given that the study was a proof-of-concept. We are currently performing some preliminary interviews with manual wheelchair users, as a better understanding of their psychological challenges in the urban context will better inform future design iterations of the prototype.

There is also room for improving the biofeedback-based intervention prototype. Based on the participants' comments regarding latency issues, future versions of the prototype need to evaluate if real-time feedback could be sufficient for a small period, and then followed by leading feedback as discussed in [23]. In addition, exploring different types and qualities of sound feedback and their psychological effects might help improve the design.

This study has helped to identify some of the main design aspects that would need to be considered and modified when adapting a biofeedback relaxation tool to the context in which it is being implemented (both protocol and prototype wise). More importantly, it draws the attention towards the under researched presented problem, and the potential that technology could have on improving the emotional experience of navigating the urban landscape in a wheelchair.

\section{REFERENCES}

[1] C. S. Holloway, A. Symonds, T. Suzuki, A. Gall, et al, "Linking wheelchair kinetics to glenohumeral joint demand during everyday accessibility activities," in Proceedings of the Annual International Conference of the IEEE Engineering in Medicine and Biology Society, EMBS, 2015, vol. 2015-Novem, pp. $2478-2481$.

[2] R. Ramirez Herrera, B. Momahed Heravi, G. Barbareschi, et al, "Towards a Wearable Wheelchair Monitor: Classification of Push Style Based on Inertial Sensors at Multiple Upper Limb Locations," in 2018 IEEE Int. Conf. on Systems, Man, and Cybernetics (SMC), 2018, pp. 1535-1540.

[3] M. Gaete-Reyes, "Citizenship and the embodied practice of wheelchair use," Geoforum, vol. 64, pp. 351-361, Aug. 2015.

[4] R. Velho, "Transport accessibility for wheelchair users: A qualitative analysis of inclusion and health," Int. J. Transp. Sci. Technol., vol. 8, no. 2, pp. 103115, Jun. 2019

[5] B. Yu, M. Funk, J. Hu, Q. Wang, and L. Feijs, "Biofeedback for Everyday Stress Management: A Systematic Review," Front. ICT, vol. 5, no. SEP, Sep. 2018.

[6] P. Paredes et al., "Just Breathe: In-Car Interventions for Guided Slow Breathing," Proc. ACM Interactive, Mobile, Wearable Ubiquitous Technol., vol. 2, no. 1, pp. 1-23, 2018.

[7] L. A. MacDonald, F. Oprescu, and B. M. Kean, "An evaluation of the effects of mindfulness training from the perspectives of wheelchair basketball players," Psychol. Sport Exerc., vol. 37, no. July 2017, pp. 188-195, 2018.

[8] L. Varvogli and C. Darviri, "Stress management techniques: Evidence-based procedures that reduce stress and promote health," Health Sc. J., vol. 5, no. 2 pp. 74-89, 2011.

[9] M. A. Russo, D. M. Santarelli, and D. O'Rourke, "The physiological effects of slow breathing in the healthy human," Breathe, vol. 13, no. 4, pp. 298-309, 2017.

[10] A. J. Mrazek et al., "The future of mindfulness training is digital, and the future is now," Curr. Opin. Psychol., vol. 28, pp. 81-86, 2019.

[11] Department for Transport, "Reported road casualties Great Britain 2017," no. September, pp. 1-376, 2018

[12] J. Eriksson, L. Girod, B. Hull, R. Newton, S. Madden, et al, "The Pothole Patrol: Using a mobile sensor network for road surface monitoring," MobiSys'08 - Proc. 6th Int. Conf. Mob. Syst. Appl. Serv., pp. 29-39, 2008

[13] J. Frey, M. Grabli, R. Slyper, and J. R. Cauchard, "Breeze: Sharing biofeedback through wearable technologies," Conf. Hum. Factors Comput Syst. - Proc., vol. 2018-April, 2018.

[14] K. Yokoyama, J. Ushida, Y. Sugiura, et al, "Heart rate indication using musical data,” IEEE Trans. Biomed. Eng., vol. 49, no. 7, pp. 729-733, Jul. 2002.

[15] A. Singh et al., "Go-with-the-Flow: Tracking, Analysis and Sonification of Movement and Breathing to Build Confidence in Activity Despite Chronic Pain," Human-Computer Interact., vol. 31, no. 3-4, pp. 335-383, 2016.

[16] M. Murgia et al., "Ecological sounds affect breath duration more than artificial sounds," Psychol. Res., vol. 80, no. 1, pp. 76-81, Jan. 2016.

[17] P. M. Lehrer and R. Gevirtz, "Heart rate variability biofeedback: how and why does it work?," Front. Psychol., vol. 5, p. 756, 2014

[18] M. Malu and L. Findlater, "Toward Accessible Health and Fitness Tracking for People with Mobility Impairments," in Proc. of the 10th EAI Int. Conf. on Pervasive Computing Tech. for Healthcare, 2016, pp. 1-8.

[19] J. W. Newbold, N. Bianchi-Berthouze, N. E. Gold, and A. Williams, "Musically informed sonification for self-directed Chronic pain physical rehabilitation," Proc. 12th Int. Conf. Sound Music Comput. SMC 2015, pp. 485-490, 2015.

[20] R. Lichenstein, D. C. Smith, J. L. Ambrose, and L. A. Moody, "Headphone use and pedestrian injury and death in the United States: 2004-2011," Inj. Prev., vol. 18 , no. 5, pp. 287-290, Oct. 2012.

[21] D. MacKenzie, B. Mortenson, and $\AA$. Brandt, "The Wheelchair Skills Program Manual," 2015.

[22] Y. Cho, S. J. Julier, and N. Bianchi-Berthouze, "Instant Stress: Detection of Perceived Mental Stress Through Smartphone Photoplethysmography and Thermal Imaging," JMIR Ment. Heal., vol. 6, no. 4, p. e10140, Apr. 2019.

[23] M. Matthews et al., "Real-time representation versus response elicitation in biosensor data," Conf. Hum. Factors Comput. Syst. - Proc., vol. 2015-April, pp. 605-608, 2015. 